\title{
Bunch Stabilization using rf Phase Modulation in the Intense Pulse Neutron Source (IPNS) Rapid Cycling Synchrotron (RCS)
}

\author{
J. C. Dooling, F. R. Brumwell, G. E. McMichael \\ Argonne National Laboratory, Argonne, IL USA
}

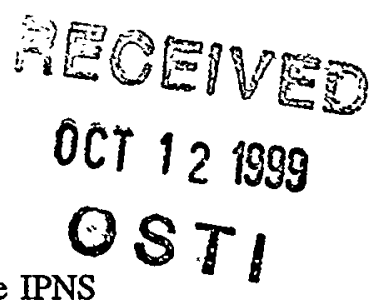

\begin{abstract}
Phase modulation (PM) is used to increase the current limit in the IPNS RCS. A device referred to as a scrambler introduces a small oscillating phase between the two RCS if cavities at approximately twice the synchrotron frequency, $\mathrm{f}_{\mathrm{s}}$. The modulation introduced by the scrambler generates longitudinal oscillations in the bunch at $2 \mathrm{f}_{\mathrm{s}}$. Modulations in the bunch are also observed transversely indicating a coupling between longitudinal and transverse motion. Comparing PM with amplitude modulation (AM), coupling to the beam is roughly equivalent at $2 \mathrm{f}_{\mathrm{s}}$.
\end{abstract}

I. Introduction.

The Intense Pulsed Neutron Source (IPNS) Rapid Cycling Synchrotron (RCS) delivers $450 \mathrm{MeV}$ protons to a heavy-metal, spallation target at $30 \mathrm{~Hz}$ with an average current of $15 \mu \mathrm{A}$. Negative hydrogen ions are injected into the RCS at $50 \mathrm{MeV}$ during a $70-80 \mu$ s period near the magnetic guide field minimum, $B_{\min }$. A carbon stripper foil converts the injected beam into protons. The frequency of the rf voltage varies from $2.21 \mathrm{MHz}$ to $5.14 \mathrm{MHz}$ during the acceleration cycle[1]. Two if cavities, located 180 degrees apart around the ring, bunch and accelerate the beam. During early operation, the achievable RCS current limit was substantially less than had been predicted. It was thought that an instability was causing the beam to grow in size transversely eventually leading it to strike the wall. Using a technique developed at KEK[2], phase modulation (PM) was added to the rf system to provide a small oscillation between the two RCS if cavities at approximately twice the synchrotron frequency. The PM device, referred to as a "scrambler," was successful in increasing the RCS current limit[3]. Prior to installation of the scrambler, the current limit was 10-11 $\mu \mathrm{A}$; with the scrambler, the current limit is now in excess of $15 \mu \mathrm{A}$. $\left(3.2 \times 10^{12} \mathrm{ppp}\right)$. The PM induces a longitudinal disturbance in the bunch, which oscillates near the scrambler frequency. The bunch oscillation couples into the perpendicular plane making it visible on transverse monitors.

\section{Longitudinal Oscillations, Instability, and Stabilization}

Fast Q signals from the beam in the RCS are detected with a toroid, then electronically assembled into a familiar "mountain range" display as shown in Figure 1. The mountain range in Figure 1a) occurs for a period just prior to the beginning of the phase modulation provided by the scrambler. The effect of the 


\section{DISCLAIMER}

This report was prepared as an account of work sponsored by an agency of the United States Government. Neither the United States Government nor any agency thereof, nor any of their employees, make any warranty, express or implied, or assumes any legal liability or responsibility for the accuracy, completeness, or usefulness of any information, apparatus, product, or process disclosed, or represents that its use would not infringe privately owned rights. Reference herein to any specific commercial product, process, or service by trade name, trademark, manufacturer, or otherwise does not necessarily constitute or imply its endorsement, recommendation, or favoring by the United States Government or any agency thereof. The views and opinions of authors expressed herein do not necessarily state or reflect those of the United States Government or any agency thereof. 


\section{DISCLAIMER}

Portions of this document may be illegible in electronic image products. Images are produced from the best available original document. 
scrambler on longitudinal bunch shape is presented in Figure 1b). The PM signal begins approximately $10 \mathrm{~ms}$ after injection when the synchrotron frequency in the bunch is approximately half the modulation frequency. The predicted synchrotron frequency (excluding space-charge) is plotted in Figure 2; also shown in this figure are synchrotron frequency data obtained with a spectrum analyzer. The measured values fall below the calculation, indicating the defocusing effect of space-charge.
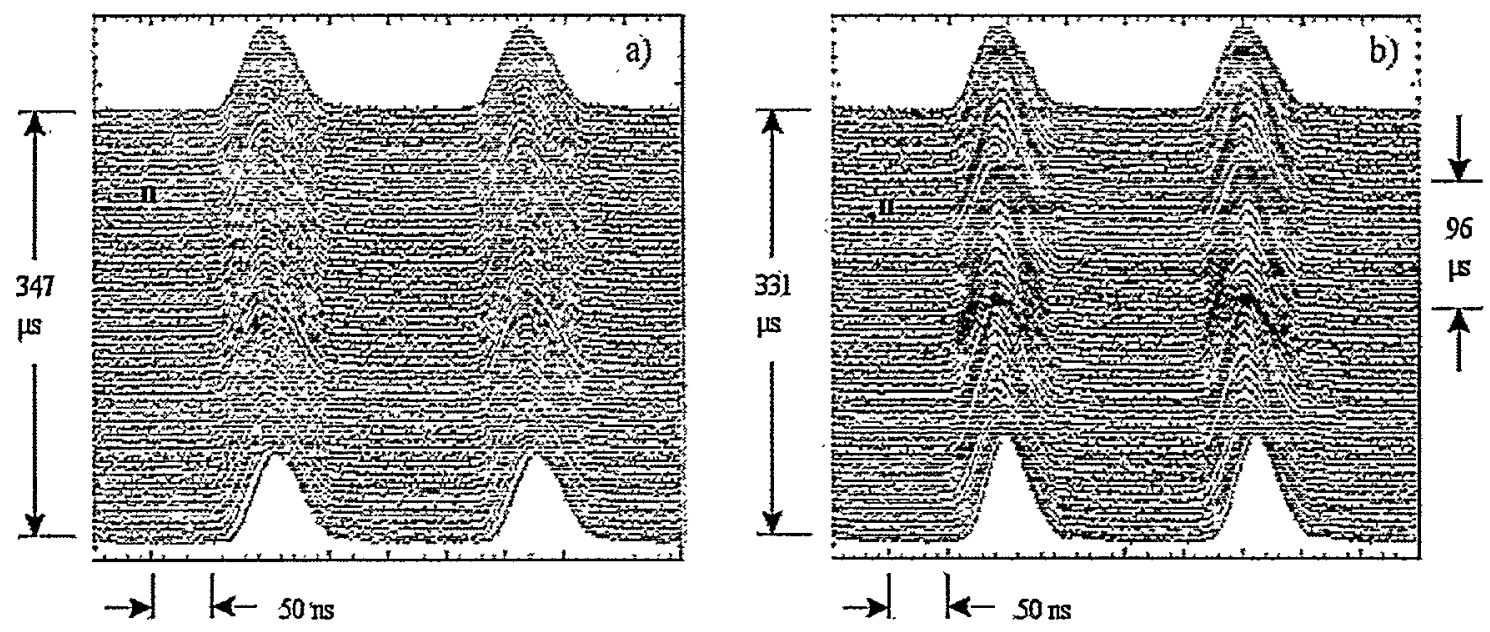

Figure 1: Mountain range display of the longitudinal bunch profiles a) before and b) during the scrambler.

The effect of the scrambler is significant with regard to both bunch dynamics and the current limit of the RCS. Without the scrambler, the maximum average current at $30 \mathrm{~Hz}$ is $10-11 \mu \mathrm{A}$; with the scrambler, in excess of $15.4 \mu \mathrm{A}$ has been achieved. The reason for the current enhancement is believed to be the result of an increased energy spread in the bunch during quadrupole oscillations. The scrambler operating at approximately twice the synchrotron frequency drives the oscillations. The increased energy spread helps to slow the growth of destructive instabilities via Landau damping. Examples of bunch profiles just prior to extraction, with and without the scrambler, are given in Figure 3. The normal quadrupolar oscillations with scrambler are in evidence in Fig. 3a); however, with the scrambler turned off, a destructive, higher-order mode is seen in Figure $3 \mathrm{~b}$ ). Losses in the latter case are significantly higher and it is not unusual to spill half the beam.

\section{Observed Transverse Effects}

As the proton beam is accelerated in the RCS, growing image currents perturb the bunch and initiate oscillations. It is known that without the scrambler, some of these oscillations grow into a destructive instability which quickly dumps 50-70 percent of the beam near the end of the cycle[3]. 


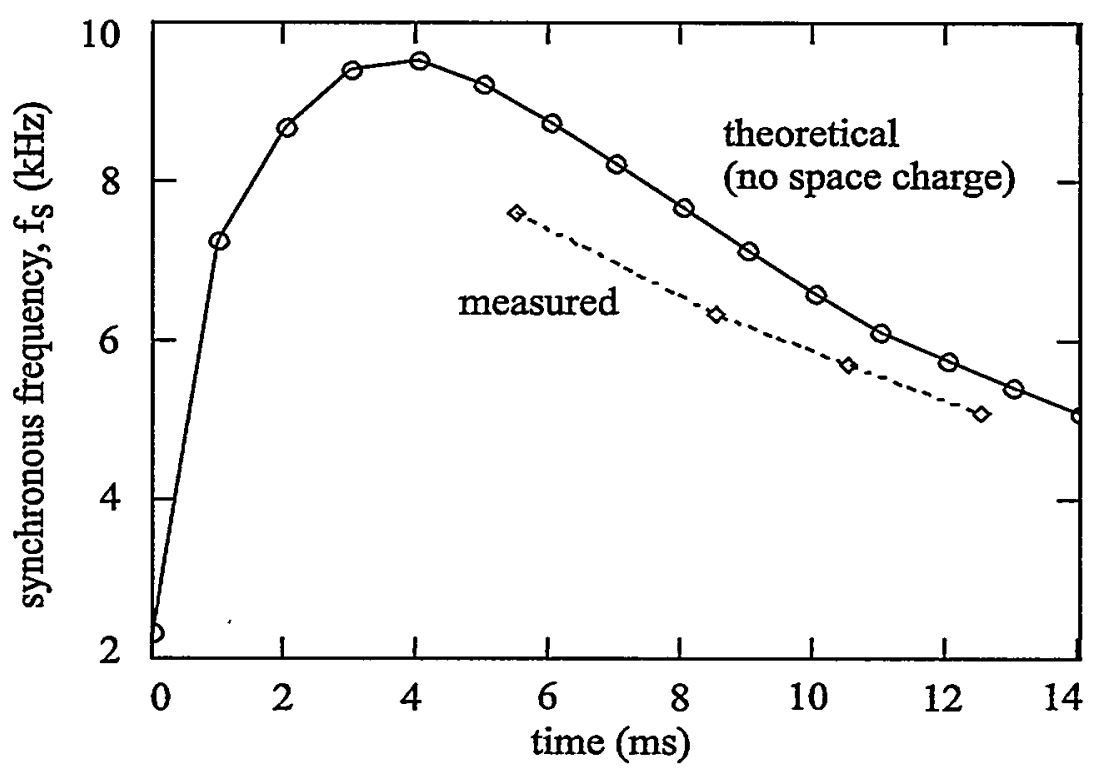

Figure 2: Synchrotron frequency in the RCS.

In addition to the scrambler, a second method is used to avoid the instability: early extraction. As indicated in Figure 4, extraction takes place approximately $2.7 \mathrm{~ms}$ prior to $B_{\max }(t=16.67 \mathrm{~ms})$. Though early extraction places greater demand on magnet supplies and rf voltage, it provides a more reliable extracted beam, by giving instabilities less time to grow. Transverse oscillations present in the RCS are most evident after initiation of the scrambler. The oscillations are observed in both loss monitors and split-ring electrode diagnostics described below.

\section{A. Split-Ring ("Pie") Electrodes.}

Split ring or "pie" electrodes are placed in a number of locations in the RCS vacuum chamber. Each pair provides horizontal or vertical beam position information. A spectral analysis of pie data has recently been performed to examine synchrotron frequencies in the RCS. A spectrum analyzer is gated to look at spectra from the pie electrodes during various periods in the RCS acceleration cycle. The gate period is held constant at $3 \mathrm{~ms}$ while the delay time is varied to cover the full range of the acceleration cycle as shown in Figure 4. Data from the spectrum analyzer is presented in Figure 5. Ostensibly, the scrambler supplies the perturbation, but in addition, discontinuities in the RCS beam pipe can act as a perturbing source via impedance mismatch and wakefield generation. Initially, the pie spectra are quiescent; however, as acceleration progresses, spectral features begin to appear close to the depressed fundamental and second harmonic synchrotron frequencies. Note that these occur prior to the scrambler. Because of the dynamic nature of the RCS, it would be desirable to examine the bunch spectra with a shorter gate. It is hoped that these tests can be conducted in the near future. 

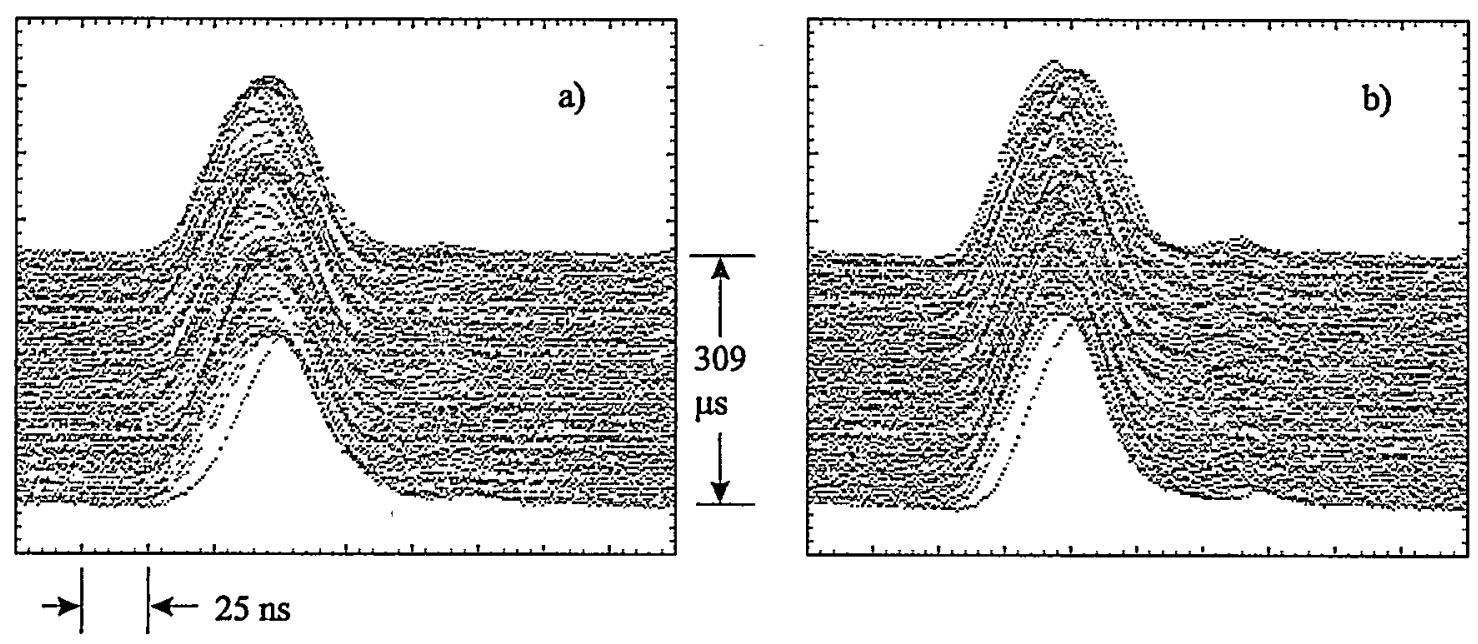

Figure 3:Mountain Range display beginning $13.55 \mathrm{~ms}$ after the end of injection, a) with the phase modulating scrambler and b) with the scrambler off.

\section{B. Loss Monitors}

The loss monitor detector front-end consists of a photomultiplier tube immersed in a bath of scintillating fluid. Loss monitors are placed at a number of locations in the RCS tunnel around the ring. After the scrambler is initiated, fluctuations are seen on the loss monitor signals. These fluctuations have a period very close to the longitudinal bunch modulations shown in Figure 1 . An example of a loss monitor signal is presented in Figure 6; also indicated in the figure are the sample periods over which FFT analyses of the data are performed.

\section{Analysis}

\section{A. Longitudinal Equations of Motion}

The use of rf voltage amplitude modulation (AM) to excite parametric oscillations in the longitudinal bunch shape has been described by $\mathrm{Li}$ et al[4]. Experimental evidence of these oscillations in the AGS was presented recently by Bai et al.[5] As mentioned above, the scrambler employs phase modulation (PM) of the if voltage to excite the RCS bunch longitudinally. In the case of the RCS, the purpose of the scrambler is to increase the momentum spread to increase Landau damping of destructive instabilities that would otherwise result in large losses from the bunch. The longitudinal equations of motion, including PM and damping, may be expressed in terms of coupled difference equations (accurate to second order) as follows,

$$
\phi_{\mathrm{n}}=\phi_{\mathrm{n}-1}+\frac{2 \pi \eta}{\beta^{2} \mathrm{E}_{\mathrm{s}}} \Delta \mathrm{E}_{\mathrm{n}-1}
$$



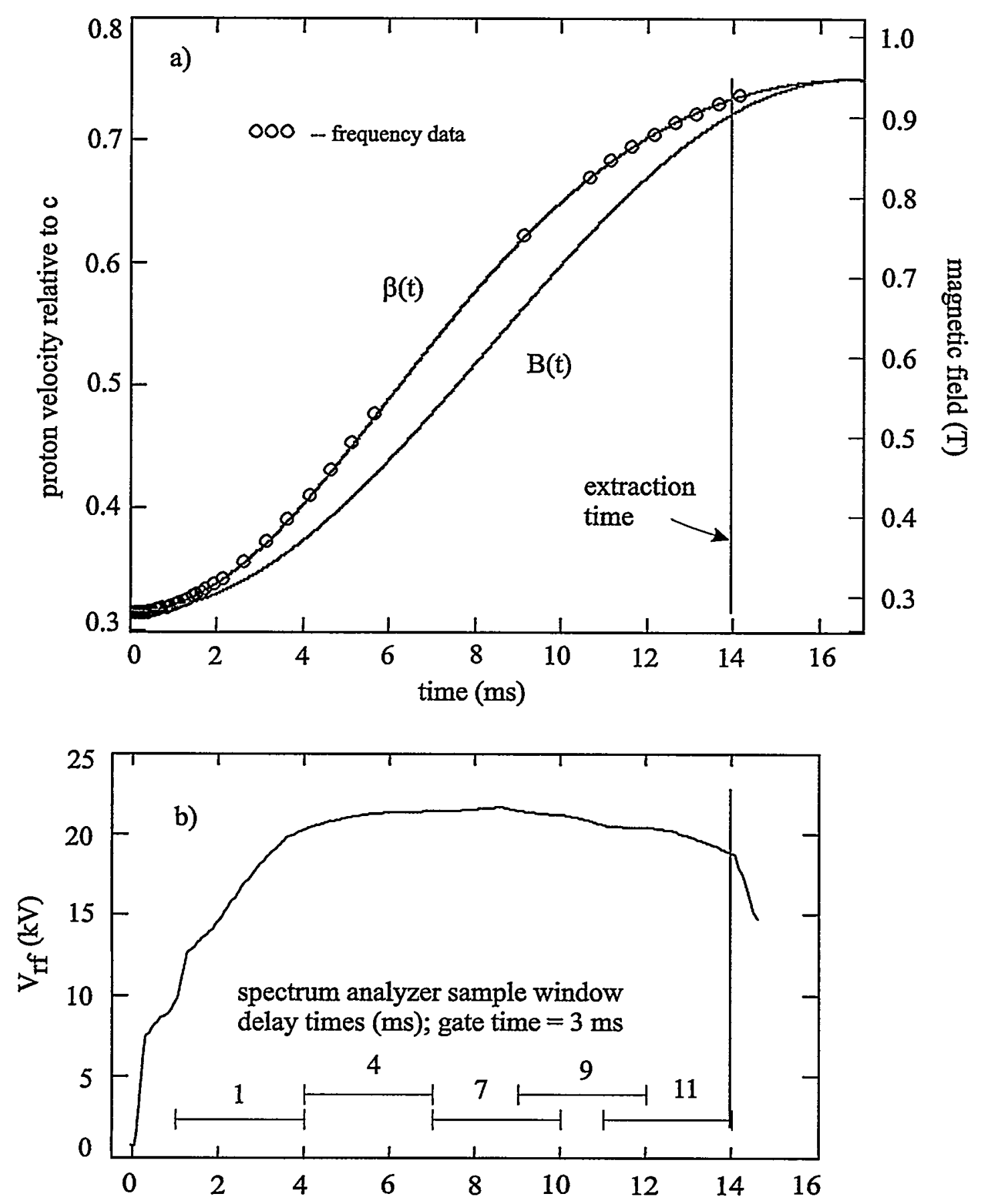

PM signal

c) time (ms)

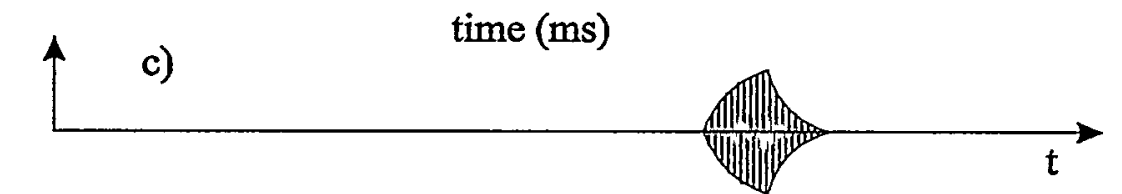

Figure 4: a) Magnetic field and proton velocity during the acceleration cycle, b) total applied RF voltage per turn with spectrum analyzer sample times on the $\mathrm{S} 5$ pie electrode indicated, and c) the scrambler pulse. 
a)

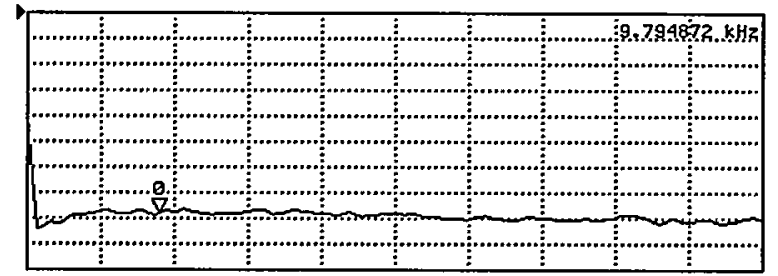

b)

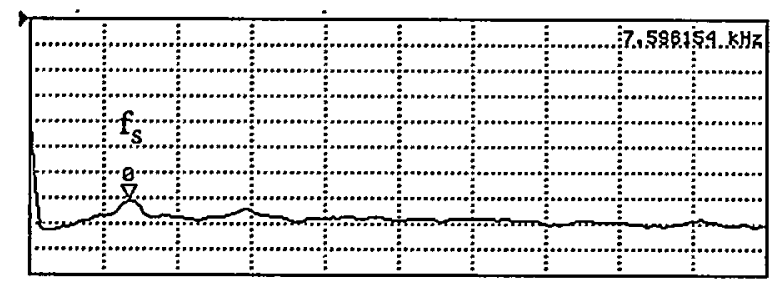

c)

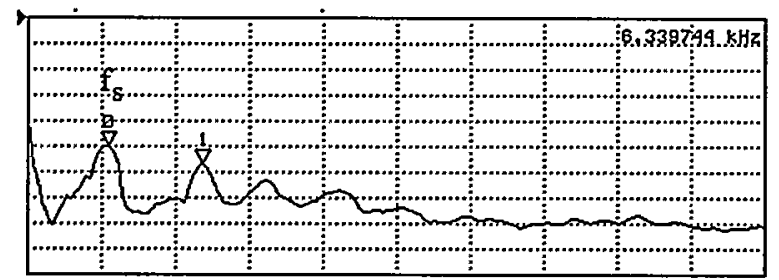

d)

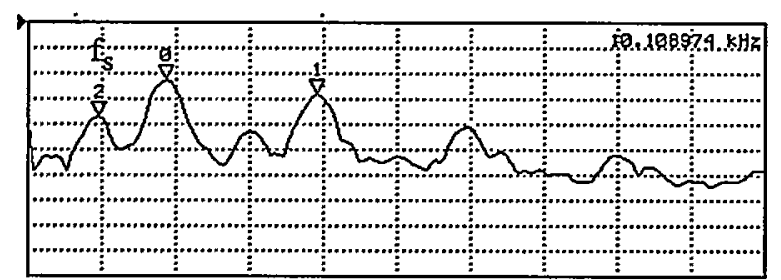

e)

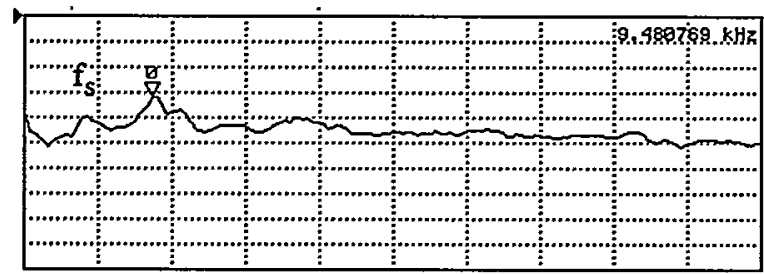

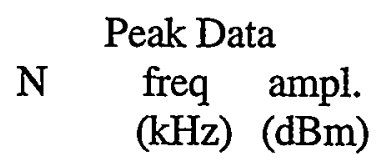

$\begin{array}{lll}0 & 7.60 & -70.9\end{array}$

$\begin{array}{lll}0 & 6.34 & -50.2\end{array}$

$1 \quad 12.62 \quad-56.9$

$\begin{array}{lll}0 & 10.11 & -22.6\end{array}$

$1 \quad 20.16-28.1$

$\begin{array}{lll}2 & 5.71 & -36.9\end{array}$

$\begin{array}{lll}0 & 9.48 & -32.2\end{array}$

Figure 5: Spectrum Analyzer signals from horizontal split ring (or "pie") electrodes in the RCS, where a) through e) correspond to delay times of 1,4 , 7,9 , and $11 \mathrm{~ms}$ after injection, respectively; the gate width is $3 \mathrm{~ms}$ (see Fig. 4). Vertical axis: $10 \mathrm{~dB} /$ div.; Horizontal axis: start freq.: $1 \mathrm{kHz}$, stop freq.: $50 \mathrm{kHz}$. The approximate location of the synchrotron frequency is indicated. 


$$
\Delta E_{n}=\left(1-\frac{4 \pi \alpha}{\omega_{r f}}\right) \Delta E_{n-1}+q V\left(\sin \left[\phi_{n}+\varepsilon \sin \left(v_{m} f_{s} \tau_{n}+\theta\right)\right]-\sin \phi_{s}\right)
$$

where $\alpha$ is the damping rate, $\omega_{\mathrm{rf}}$ is the rf radian frequency, $v_{\mathrm{m}}$ is harmonic number of the modulation relative to the synchrotron tune, $v_{\mathrm{s}}$ (i.e., for second harmonic PM, $v_{m}=2$ ), the synchrotron frequency $f_{s}=v_{s} f_{r f}, \tau_{n}=n / f_{r f}$, and $\eta$ is the slip factor. The synchrotron tune is defined as,

$$
v_{s}=\sqrt{\frac{h|\eta| q V}{2 \pi \beta^{2} E_{s}}}
$$

Equations 1 and 2 introduce PM through the voltage term rather than directly in the particle phase equation since bunch motion is affected through the applied field. The IPNS RCS accelerates a single bunch so the harmonic number, $\mathrm{h}=1$. Acceleration is implied by a nonzero $\phi_{s}$; however, it is assumed that the change in $\beta$ and $E_{s}$ are small during one rf cycle. Using the longitudinal analysis described above, the effects of $\mathrm{PM}$ on phase-space trajectories are investigated. Figure 7 presents a comparison of comparable $\mathrm{AM}\left(\varepsilon_{\mathrm{AM}}=0.018\right.$, analysis not presented) with $\mathrm{PM}\left(\varepsilon_{\mathrm{PM}}=0.02 \mathrm{rad}\right)$ near the second harmonic of the synchrotron frequency. In both cases, damping is neglected $(\alpha=0)$. The modulation depth for AM is made equivalent to a 0.02 radian shift in PM, equivalent to the value presently used by the RCS scrambler at a synchronous phase angle of 0.472 radians; i.e., $\Delta V / V=\Delta \phi \cos \left(\phi_{s}\right)=0.018$.

\section{B. Transverse Coupling}

The appearance of both transverse and longitudinal oscillations at roughly the same frequency indicates a coupling between longitudinal and transverse motion in the bunch. Such a coupling can arise from intrabeam scattering caused by space-charge. Scattering drives both longitudinal and transverse temperatures toward equilibrium. If one assumes that the total energy in the bunch is conserved, then one can write[6],

$$
\gamma \mathrm{mv_{ \textrm {x } } ^ { 2 }}+\gamma \mathrm{mv_{ \textrm {y } } ^ { 2 }}+\gamma^{3} \mathrm{~m} \overline{\left(\Delta \mathrm{v}_{\mathrm{z}}\right)^{2}}=\mathrm{K}
$$

where $\mathrm{K}$ is a constant. Assume, for simplicity, a $\mathrm{KV}$ distribution in four-dimensional transverse phase space $^{7}$,

$$
\frac{\mathrm{a}^{2}}{\mathrm{x}^{2}}+\left(\frac{\mathrm{ax}}{\varepsilon_{\mathrm{x}}}\right)^{2}+\frac{\mathrm{b}^{2}}{\mathrm{y}^{2}}+\left(\frac{\mathrm{by^{ \prime }}}{\varepsilon_{\mathrm{y}}}\right)^{2}=1
$$




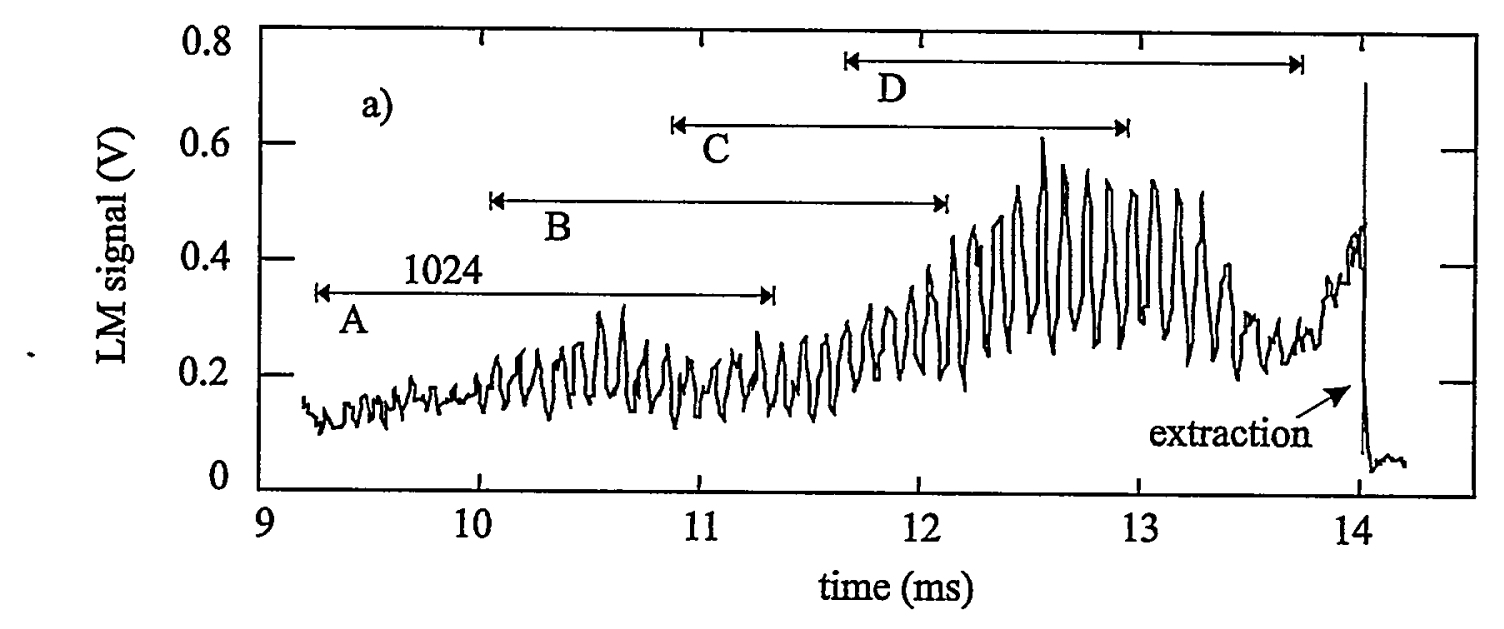

b)
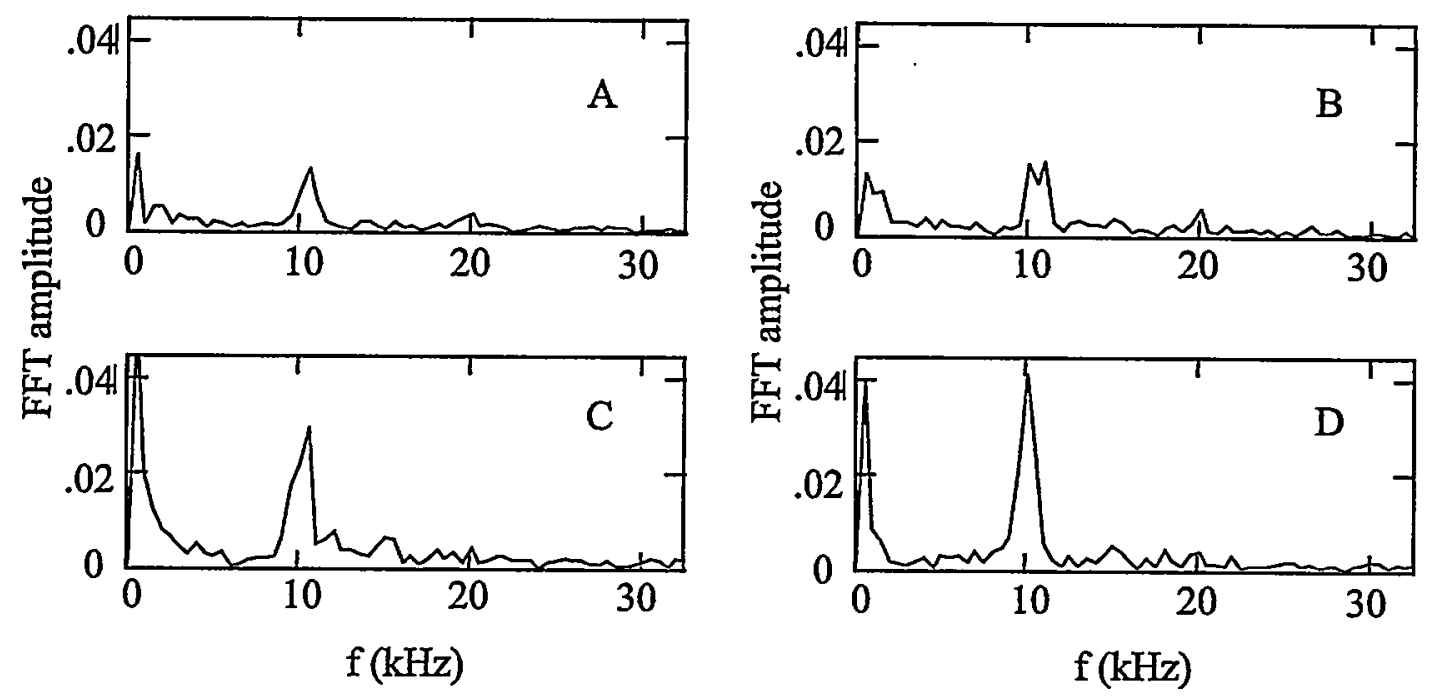

Figure 6: a) Loss monitor data indicating sample windows for 1024-word FFT and b) selected FFT spectra for times shown in a).

where the $\mathrm{x}$ and $\mathrm{y}$ beam sizes and emittances are $\mathrm{a}, \mathrm{b}, \varepsilon_{\mathrm{x}}$, and $\varepsilon_{\mathrm{y}}$, respectively; and $x^{\prime}=v_{x} / v_{z}$ and $y^{\prime}=v_{y} / v_{z}$. As PM excites a quadrupolar oscillation in longitudinal phase space (as suggested from Figs. 1 and 2), $\Delta \mathrm{E}$ and hence the average of $\left(\Delta \mathrm{v}_{\mathrm{z}}\right)^{2}$ oscillate as well. Since total energy is conserved, the average perpendicular energy must vary in accordance with Eq. 4. If transverse emittances or $x^{\prime}$ and $y^{\prime}$ vary, then to satisfy Eq. 5 , it is reasonable to expect transverse beam sizes will also be affected. Measured transverse emittance in the beam extracted from the RCS does show an increase with injected charge or circulating current, suggesting that space-charge induced intrabeam scattering may be at work; however, other factors may also contribute to the rise in emittance. 


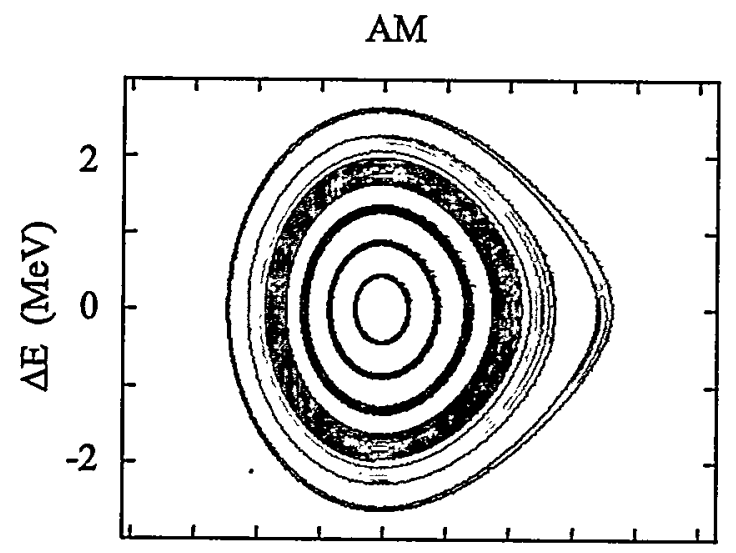

PM

a)
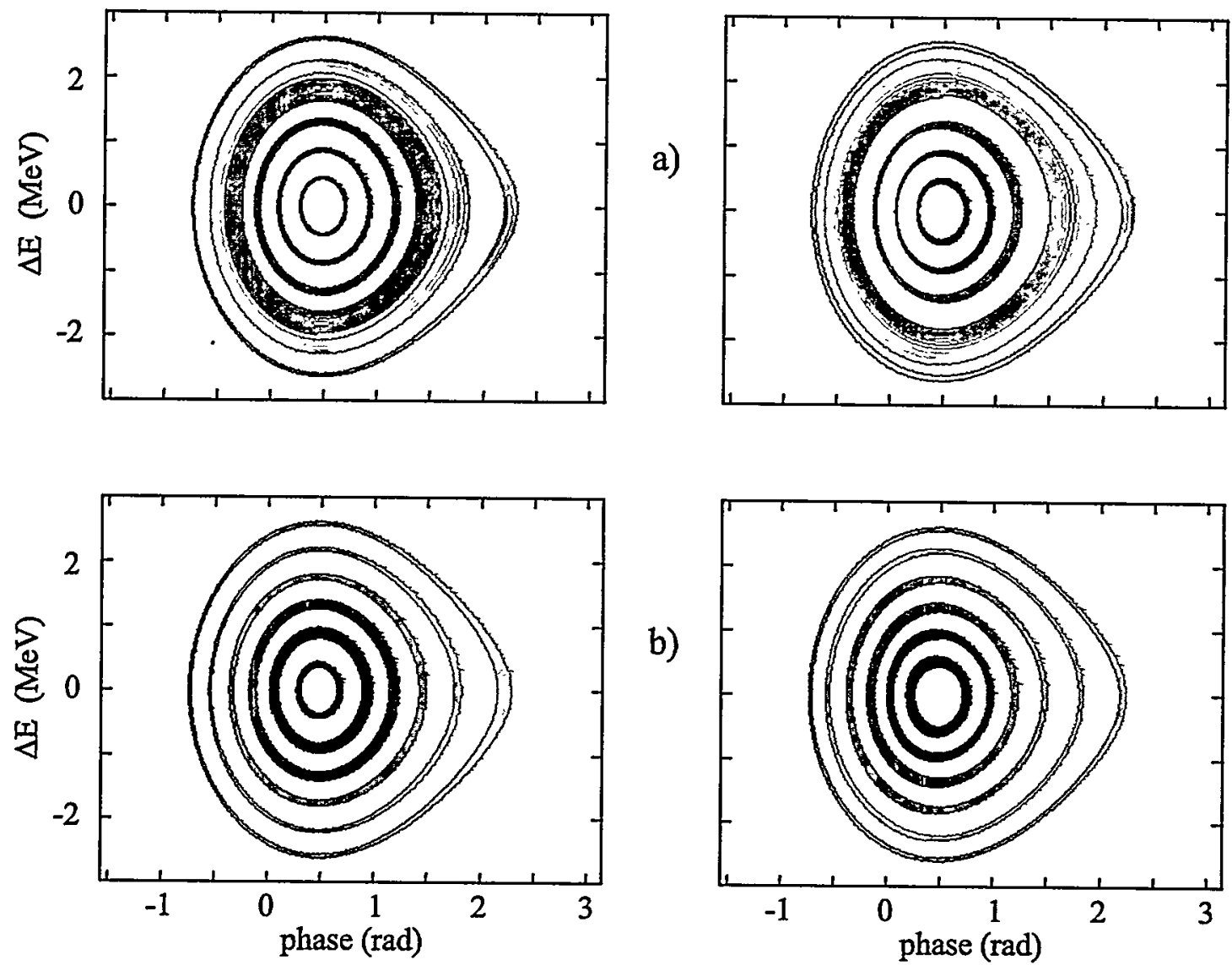

Figure 7: Comparison of six AM (left) and PM (right) phase-space trajectories for a) $v_{m}=1.8$ and b) $v_{m}=2.0$, with $\varepsilon_{A M}=0.018, \varepsilon_{P M}=0.02 \mathrm{rad}, f_{s}=6.6 \mathrm{kHz}$, and $\phi_{\mathrm{s}}=0.472 \mathrm{rad}$. The starting phase-space coordinates of the six trajectories are repeated in each of the four cases.

\section{Discussion}

A figure of merit for synchrotrons is the charge per phase-space that can be accelerated successfully. In the RCS, the peak phase-space-charge density occurs $7 \mathrm{~ms}$ after injection at a value of $11.0 \times 10^{12}$ protons $/ \mathrm{eV}$-sec $\left(3.2 \times 10^{12} \mathrm{p} / 0.29 \mathrm{eV}\right.$-sec). The high space-charge phase density leads to a substantial reduction of the synchrotron frequency in the bunch as indicated in Figure 2.

From the preliminary analysis presented here, the RCS bunch appears to have a synchrotron frequency just above half that of the scrambler when the PM signal begins. The second harmonic initially couples to the outermost edges of the bunch where the local synchrotron frequency is lowest. As time progresses and the synchrotron frequency decreases, the oscillation moves toward the inner phase- 
space of the bunch. This effect is illustrated in Figure 7 for both AM and PM. For both types of modulation, the effect on the outer trajectories is roughly equivalent. The modulation varies the energy amplitude of the trajectories within the stable phase-space of the bucket and would presumably extend to all charged particles within this band of phase-space in the bunch.

Presently, we are adding AM and PM to the CAPTURE_SPC analysis $[8,9]$ to determine if quadrupolar oscillations in the bunch can be generated when spacecharge and acceleration are included. In addition, these effects will be examined in the presence of second harmonic $\operatorname{rf}[10]$.

\section{Acknowledgements}

The authors wish to thank Dr. S. Y. Lee for his helpful comments and the IPNS Operations Staff for their continued dedication and support.

This work is supported by the United States Department of Energy under contract no.: W-31-109-ENG-38.

\section{References}

[1] T. W. Hardek, and F. E. Brandeberry, IEEE Trans. Nuc. Sci., 26(3), 3021(1979).

[2] Y. Mizumachi and K. Muto, IEEE Trans. Nuc. Sci., 28(3), 2563(1981).

[3] C. Potts, et al., IEEE Trans. Nuc. Sci., 32(5), 3107(1985).

[4] Li et al., Nuc. Instrum. Meth., 364 A, 205(1995).

[5] M. Bai, et al., Proc. of the 1999 Particle Accelerator Conf., New York, March 28-April 2, 1999

[6] M. Reiser, Theory and Design of Charged Particle Beams, Wiley, New York, 1994, p. 531.

[7] Ibid, p. 61.

[8] Y. Cho, E. Lessner, K. Symon, Proc. European Particle Accelerator Conf., p. 1228, (1994).

[9] E. Lessner and K. Symon, Computational Accelerators Physics, Williamsburg, Virginia, September 1996, AIP Conf. Proc. 391, p.185 (1997).

[10] J.C.Dooling, et al., Proc. of the 1999 Particle Accelerator Conf. New York, March 28-April 2, 1999 